\title{
Plasmon dispersion in graphite: A comparison of current ab initio methods
}

\author{
Sean M. Anderson, ${ }^{1,{ }^{*}}$ Bernardo S. Mendoza, ${ }^{1}$ Giorgia Fugallo, ${ }^{2}$ and Francesco Sottile ${ }^{3,4}$ \\ ${ }^{1}$ Centro de Investigaciones en Óptica, León, Guanajuato, México \\ ${ }^{2}$ CNRS, UMR 6607, Laboratorie de Thermique et Energie de Nantes (LTeN) Polytech'Nantes, Université de Nantes, \\ Rue Christian Pauc, F-44306 Nantes Cedex 3, France \\ ${ }^{3}$ Laboratoire des Solides Irradiés, École Polytechnique, CNRS, CEA, Université Paris-Saclay, F-91128 Palaiseau, France \\ ${ }^{4}$ European Theoretical Spectroscopy Facility (ETSF)
}

(Received 10 December 2018; revised manuscript received 9 May 2019; published 22 July 2019)

\begin{abstract}
We perform a systematic study of the macroscopic dielectric function and electron energy loss (EEL) spectra for graphite. We obtain the dispersion behavior for the $\pi$ plasmon, as a function of the momentum transfer $q$ for two nonequivalent paths that traverse the first four Brillouin zones. We carry out these calculations within both time-dependent density functional theory (with two exchange-correlation functionals) and the Bethe-Salpeter equation. Additionally, we explore the effects of using the complete excitonic Hamiltonian (with all electronhole pairs and antipairs), and within the Tamm-Dancoff approximation (neglecting antipairs). By analyzing the behavior of the macroscopic dielectric function, we are able to determine which peaks are predominantly from plasmonic behavior or only interband transitions. We compare the calculated spectra against several experiments that span almost five decades; our results present clear trends that follow the physical origins of the observed peaks. We carry out this study over a large range of momentum transfer in order to better evaluate the different theoretical methods compared to experiment, and predict the plasmonic behavior beyond available experimental data. Our results indicate that including the complete Hamiltonian with the exciton coupling included is essential for accurately describing the observed EEL spectra and plasmon dispersion of graphite, particularly for low values of momentum transfer. However, the solution of the Bethe-Salpeter equation is computationally intensive, so time-dependent density functional theory methods used in conjunction with the complete Hamiltonian may be an attractive alternative.
\end{abstract}

DOI: 10.1103/PhysRevB.100.045205

\section{INTRODUCTION}

The study of the optical spectra of solids yields important insight into the underlying electronic and structural properties of a material. When an incident photon is absorbed by a material, the system is energetically excited from its ground to an excited state, forming an electron-hole pair or exciton, a localized quantum of neutral electron-hole (e-h) pairs bound by the Coulomb attraction. From a theoretical standpoint, $a b$ initio calculations are essential tools for elucidating these excited-state electronic properties of solids, surfaces, and nanostructures. Ground-state properties can be calculated accurately using density functional theory (DFT) [1,2], but the aforementioned electronic excitations necessitate further theoretical developments. Time-dependent density functional theory (TDDFT) [3] is a natural extension of DFT, and is able to successfully describe spectra like electron energy loss spectroscopy (EELS) or inelastic x-ray scattering of simple semiconductors, as well as the photoabsorption cross section of simple molecules. However, while the use of the local density approximation (LDA) in DFT yields qualitative (and more often than not, quantitative) agreement with experiment for the ground-state properties of many materials, the same cannot be said for using the adiabatic LDA (ALDA)

*sma@cio.mx functional [3] in TDDFT (the TDDFT equivalent of DFTLDA). This functional does not consistently improve the calculated optical spectra of solids with respect to the common random phase approximation (RPA), where the exchangecorrelation functional, the main ingredient of TDDFT, is neglected. It is in fact well known [4] that today functionals and approximations to TDDFT are not well suited for accurately calculating the absorption spectra of most solids [4,5], where excitonic behavior predominates.

Thus, the behavior of complex electronic excitations can only be accurately described by making use of many-body perturbation theory (MBPT) [6]. In particular, using Hedin's $G W$ approximation [7] followed by solving the Bethe-Salpeter equation (BSE) [4,8-11] allows for the accurate calculation of the optical properties of many physical systems, within a completely $a b$ initio framework [12-23]. In general, absorption and scattering spectroscopies (that concern neutral excitations) are very well described by the BSE [4,24-27]. Within this $G W / \mathrm{BSE}$ approach, excitons are described as a combination of e-h pairs of a noninteracting system; we can convert the problem to an effective eigenvalue problem in the e-h basis. However, nanoscale materials involve a huge number of e-h pairs, which makes solving the BSE extremely costly from a computational standpoint. The Tamm-Dancoff approximation (TDA) [6] is an approximation that only considers positive-energy e-h pairs, effectively neglecting the interaction between e-h pairs at positive and negative (antipairs) 
energies. The non-Hermitian BS problem is thus reduced to a Hermitian problem that can be solved with efficient and stable iterative methods. The Haydock recursion scheme [28-30] is one of the most efficient and computationally inexpensive iterative methods used for dealing with the Hamiltonian. Thanks to this reduction in the computational complexity, the TDA has been applied to a variety of systems [31-33].

Excitations from plasmons, collective oscillations of the electronic density that induce a macroscopic polarization, can also feature very prominently in the measured EEL spectra for some materials. These oscillations involve the creation of e-h antipairs; thus, these plasmonic features are inherently ill described by the TDA [34-37]. Accurately describing both plasmonic and excitonic features is essential if we wish to accurately characterize and analyze the system; therefore, we require a suitable test case in order to carry out a complete study of these theoretical methods. Optimally, this reference material will display plasmonic behavior, be theoretically and experimentally well characterized, and have continued relevance in current research. We consider that bulk graphite meets these requirements and can make for a very effective benchmark. Early work revealed accurate band structure calculations $[38,39]$ that continue to be experimentally studied by very precise photoemission and ARPES experiments [40,41]. A great variety of EELS [42-47] and absorption [47-49] measurements have been carried out with relevant theoretical developments, including detailed analysis of the low-energy $(\pi)$ and high-energy $(\pi+\sigma)$ plasmons. Concerning the actual plasmon dispersion, there is very recent work $[50,51]$ featuring very high resolution EELS measurements for a variety of values of momentum transfer. Lastly, graphite continues to be of relevance as the precursor of graphene, which presents its own unique spectroscopic characteristics [51-56], many of which can be explained through its relationship with graphite.

Therefore, our motivation for this study is to compare the available theoretical frameworks and apply them towards a reference material, graphite, with a solid body of consistent experimental characterization behind it. We perform a systematic study of the macroscopic dielectric function and EEL spectra for graphite, in order to elucidate the $\pi$-plasmon dispersion behavior as a function of the momentum transfer $q$ for two nonequivalent paths that encompass the first four Brillouin zones. We carry out these calculations within both TDDFT (with two exchange-correlation functionals) and the BSE; additionally, we explore the effects of using the complete excitonic Hamiltonian (with all e-h pairs and antipairs), and within the TDA (neglecting antipairs). The resulting spectra are consistent with previous results featured in the literature, and we can accurately discern which peaks derive from plasmonic behavior or from interband transitions. However, as each method considers very different approximations, the peak positions and intensity change substantially. We study these characteristics and compare them against several experiments that present consistent tendencies, even though they were carried out in different periods and by different groups. Our calculated spectra present clear trends that follow the physical origins of the observed peaks. Given the large momentum transfer values (up to $q=3.22 \AA^{-1}$ ), and the comparison of several methods with various experiments, we consider this to be a thorough benchmark for future reference.
This paper is organized as follows. In Sec. II, we present the theoretical framework that describes the aforementioned methods. In Sec. IV, we present our results for the $q$ dependent plasmon dispersion over two separate, nonequivalent paths in the Brillouin zone, and show several detailed comparisons with experiment. We list our conclusions and final remarks in Sec. V.

\section{THEORY}

In order to better explain the differences between the TDDFT and BSE frameworks, we will describe them in the same formalism for clarity and ease of interpretation. The key quantity measured in absorption and EELS is the macroscopic dielectric function, $\epsilon_{\mathrm{M}}$; specifically, $\operatorname{Im} \epsilon_{\mathrm{M}}$ is measured in absorption experiments, while $-\operatorname{Im}\left(1 / \epsilon_{\mathrm{M}}\right)$ is measured in EELS. The macroscopic dielectric function is connected to the inverse dielectric function $\epsilon^{-1}$ as

$$
\epsilon_{\mathrm{M}}(\omega) \equiv \lim _{\mathbf{q} \rightarrow 0} \frac{1}{\left[\epsilon^{-1}(\mathbf{q}, \omega)\right]_{\mathbf{G}=\mathbf{G}^{\prime}=0}},
$$

where $\mathbf{G}$ and $\mathbf{G}^{\prime}$ are reciprocal lattice vectors. We can express $\epsilon^{-1}$ for both TDDFT and the BSE as a Dyson-like equation,

$$
D=D^{(0)}+D^{(0)} K D .
$$

For TDDFT, $D$ is the two-point polarizability $\chi$, from which we can obtain the inverse dielectric function $\epsilon^{-1}=1-v \chi(v$ is the Coulomb potential); for the BSE, $D$ is the two-particle correlation function $L$ which yields $\chi$ by contracting two of its four indices,

$$
\chi(1,2)=L\left(1,1^{+}, 2,2^{+}\right),
$$

where (1) is shorthand notation for specific position, time, and spin states.

The two theories can be recast in the same equation, but this similarity in form hides some key differences [57,58]:

(1) TDDFT leads to two-point equations for describing the propagation of the density; BSE describes the propagation of an electron (e) and a hole (h), and thus leads to four-point equations [as evident from Eq. (2)].

(2) In TDDFT, $D^{(0)}$ is the independent-particle response function $\chi^{(0)}$ constructed with the Kohn-Sham (KS) orbitals and eigenvalues; in the BSE formalism, $D^{(0)}$ is the independent quasiparticle response $L^{(0)}$ constructed using quasiparticle eigenvalues and eigenfunctions (obtained, for example, via a $G W$ calculation as done in this work).

(3) In TDDFT, the kernel $K=v+f_{x c}$, where $f_{x c}$ is the functional derivative with respect to the density of the exchange-correlation potential $v_{x c}$, and so $f_{x c}=\delta v_{x c} / \delta \rho$. For the BSE, $K=v-W$ with $W$ the screened version of the bare Coulomb interaction $v$.

Regarding this last point, different approximations are possible for $v_{x c}$ and thus $f_{x c}$. In this work, we will compare the adiabatic local density approximation (ALDA), where the exchange-correlation potential is taken in the LDA,

$$
f_{x c}^{\mathrm{ALDA}}=\delta v_{x c}^{\mathrm{LDA}} / \delta \rho,
$$

and the random phase approximation (RPA), where

$$
f_{x c}^{\mathrm{RPA}}=0 .
$$


In the case of the BSE, the screening term $W$ is commonly taken in a static version calculated at the RPA level $[4,57]$. While $v$ enters in the kernel $K$ as a repulsive e-h exchange interaction and is responsible for the local-field effects, the second term in both theories $\left(f_{x c}\right.$ or $\left.-W\right)$ describes the attractive interaction which is the origin of the excitonic effects, including the formation of bound excitons.

In order to obtain a spectral representation of $D$ (given that only a small number of transitions will contribute to each part of the spectrum), it is useful to reformulate the Dyson-like Eq. (1) as an eigenvalue problem $[4,34,59,60]$ by introducing an effective two-particle excitonic Hamiltonian, $H_{\mathrm{exc}}$, thus obtaining an eigenvalue problem,

$$
H_{\text {exc }}\left(\mathbf{q}_{\mathbf{r}}\right) A_{\lambda}\left(\mathbf{q}_{\mathbf{r}}\right)=E_{\lambda}\left(\mathbf{q}_{\mathbf{r}}\right) A_{\lambda}\left(\mathbf{q}_{\mathbf{r}}\right) .
$$

The excitonic Hamiltonian is written in a basis of electronhole transitions $\left(n_{1} k_{1} \rightarrow n_{2} k_{2}\right)$. These transitions can be classified as resonant transitions, $\left(v, k-q_{r}\right) \rightarrow(c, k)$, or antiresonant transitions, $(c, k) \rightarrow\left(v, k+q_{r}\right)$, depending on whether the band index $n$ is associated with an occupied valence $(v)$ band or an unoccupied conduction $(c)$ band. Lastly, $q_{r}$ is a momentum transfer belonging to the first Brillouin zone [23]. The excitonic Hamiltonian has a block matrix form $[22,23,25,36]$,

$$
H_{\mathrm{exc}}=\left(\begin{array}{cc}
R & C^{R, A} \\
C^{A, R} & A
\end{array}\right) .
$$

When working in the long-wavelength limit $\left(q_{r} \rightarrow 0\right), A=$ $-R^{*}$ and $C^{A, R}=-\left[C^{R, A}\right]^{*}$. The diagonal $A$ and $R$ blocks are Hermitian, while the coupling $C$ blocks are symmetric. When dealing with a generic momentum transfer $q_{r} \neq 0$ then $A \neq-R^{*}$ and the coupling terms are no longer symmetric, effectively doubling the computational cost of evaluating $H_{\text {exc }}$.

By taking advantage of the fact that the off-diagonal terms are typically significantly smaller than the resonant terms, it is possible to reduce the computational cost by using the Tamn-Dancoff approximation (TDA) [6] by setting the off-diagonal coupling terms $C$ to zero. In other words, the interaction between e-h pairs at positive and negative (antipairs) energies is neglected, and only one e-h pair is assumed to propagate in any time interval. Considering the different nature and locality of excitons and plasmons, it is clear that the TDA is more unreliable for describing plasmons, where the density oscillations involve the excitation of large numbers of e-h antipairs. Nevertheless, due to the success obtained in describing the optical absorption of solids and thanks to the remarkable numerical advantages, the TDA has been applied to many different systems [57]. Under the TDA the Hamiltonian becomes a Hermitian operator, enabling the use of efficient iterative schemes for solving the BSE such as the Haydock recursion method [15,22,28-30,61].

The advantage of having the spectral representation of the excitonic Hamiltonian is clear; instead of inverting a matrix for each frequency, we are only required to diagonalize it once for all [60]. Regardless of the framework (TDDFT or BSE) or the method used for diagonalizing the excitonic Hamiltonian, it is possible to obtain the macroscopic dielectric function from the eigenvalues $\left(E_{\lambda}\right)$ and eigenstates $\left(A_{\lambda}\right)$ [4]; this is then used to calculate the theoretical spectra that can be directly compared with experimental data. The general expression for
TABLE I. Naming scheme employed for the calculations featured in this work.

\begin{tabular}{lcccc}
\hline \hline \multicolumn{2}{c}{ Label } & Type & Hamiltonian & $f_{x c}$ \\
\hline BSE & CP/TD & MBPT & Full/Tamm-Dancoff & \\
ALDA & CP/TD & TDDFT & Full/Tamm-Dancoff & $\delta v_{x c}^{\text {LDA }} / \delta \rho$ \\
RPA & CP/TD & TDDFT & Full/Tamm-Dancoff & 0 \\
\hline \hline
\end{tabular}

$$
\begin{aligned}
& \epsilon_{M} \text { is } \\
& \epsilon_{M}(\omega) \\
& \quad=1-\lim _{\mathbf{q} \rightarrow 0} v_{0}(\mathbf{q}) \sum_{\lambda, \lambda^{\prime}}\left[\sum_{\left(n_{1}, n_{2}\right)}\left\langle n_{1}\left|e^{-i \mathbf{q} \cdot \mathbf{r}}\right| n_{2}\right\rangle \frac{A_{\lambda}^{\left(n_{1}, n_{2}\right)}}{E_{\lambda}-\omega-i \eta} S_{\lambda, \lambda^{\prime}}^{-1}\right. \\
& \left.\quad \times \sum_{\left(n_{3}, n_{4}\right)}\left\langle n_{4}\left|e^{i \mathbf{q} \cdot \mathbf{r}^{\prime}}\right| n_{3}\right\rangle A_{\lambda}^{*\left(n_{3}, n_{4}\right)}\left(f_{n_{4}}-f_{n_{3}}\right)\right],
\end{aligned}
$$

with occupation functions $f_{n_{i}}$, long-range component of the Coulomb potential $v_{0}$, and overlap matrix

$$
S_{\lambda, \lambda^{\prime}}=\sum_{n_{1} n_{2}} A_{\lambda}^{*\left(n_{1} n_{2}\right)} A_{\lambda^{\prime}}^{\left(n_{1} n_{2}\right)}
$$

In the TDA, the Hamiltonian becomes Hermitian and the eigenstates $A_{\lambda}$ are mutually orthogonal. This results in a greatly reduced expression for the macroscopic dielectric function,

$\epsilon_{M}^{\mathrm{TDA}}(\omega)=1-\lim _{\mathbf{q} \rightarrow 0} v_{0}(\mathbf{q}) \sum_{\lambda} \frac{\left|\sum_{\left(n_{1}, n_{2}\right)}\left\langle n_{1}\left|e^{-i \mathbf{q} \cdot \mathbf{r}}\right| n_{2}\right\rangle A_{\lambda}^{\left(n_{1}, n_{2}\right)}\right|^{2}}{E_{\lambda}-\omega-i \eta}$.

Note that for large momentum transfer,

$$
\begin{aligned}
-\operatorname{Im} \epsilon_{\mathrm{M}}^{-1}(\mathbf{q}, \omega) & =\frac{\operatorname{Im} \epsilon_{\mathrm{M}}(\mathbf{q}, \omega)}{\left[\operatorname{Re} \epsilon_{\mathrm{M}}(\mathbf{q}, \omega)\right]^{2}+\left[\operatorname{Im} \epsilon_{\mathrm{M}}(\mathbf{q}, \omega)\right]^{2}} \\
& \approx \operatorname{Im} \epsilon_{\mathrm{M}}(\mathbf{q}, \omega),
\end{aligned}
$$

since $\operatorname{Re} \epsilon_{\mathrm{M}}(\mathbf{q}, \omega) \rightarrow 1$, and $\operatorname{Im} \epsilon_{\mathrm{M}}(\mathbf{q}, \omega)$ is very small [23]. Thus, the EEL spectra will increasingly mimic the absorption spectra for large values of $q$.

Table I enumerates the labels that we will use for the remainder of this work, and the particular method described by each label.

\section{COMPUTATIONAL DETAILS}

The electronic ground state and band structure of bulk graphite were calculated using DFT-LDA [1,2], using normconserving Troulliers-Martins pseudopotentials [62] using the plane-wave basis method implemented within the ABINIT $[63,64]$ code. Our calculated DFT band structure is identical to previously reported results [65]. We constructed the graphite structure using lattice constants $a=2.45 \AA$ and $c=6.69 \AA$, obtained from structural optimization studies. The groundstate energy was calculated using an $11 \times 11 \times 4$ MonkhorstPack grid of $\mathbf{k}$ points, with an energy cutoff of $20 \mathrm{Ha}$.

We then selected two separate k-point grids for carrying out our study of plasmon dispersion over different momentum values, $q$. These momentum vectors follow two in-plane paths along the Brillouin zones: first, from $\Gamma$ to the $M$ point of 


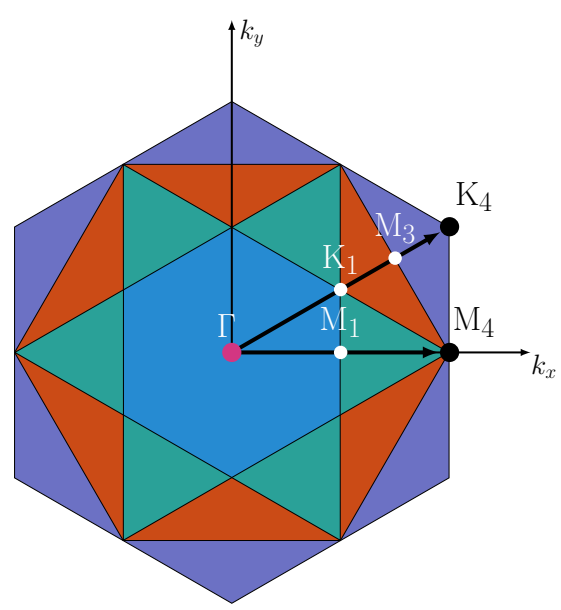

FIG. 1. First four Brillouin zones with the two selected paths (1) $\Gamma$ to $\mathrm{M}$ in the fourth zone $\left(\mathrm{M}_{4}\right)$, and (2) $\Gamma$ to $\mathrm{K}$ in the fourth zone $\left(\mathrm{K}_{4}\right)$, along which we calculate the EEL spectra and macroscopic dielectric function.

the fourth zone $\left(\mathrm{M}_{4}\right)$, and second, from $\Gamma$ to the $\mathrm{K}$ point of the fourth zone $\left(\mathrm{K}_{4}\right)$. Both paths pass through various points of symmetry; see Fig. 1 for a graphical representation. The selected $\Gamma$-centered $14 \times 14 \times 2$ (392 k points) and $12 \times 12 \times 2$ ( $288 \mathbf{k}$ points) grids were chosen due to the easy accommodation to the desired $q$ values (commonly used in experiments) along both paths, and because they offered an acceptable compromise between convergence and computational expense. For the $\Gamma \rightarrow \mathrm{M}_{4}$ path we used both grids in order to provide a more dense sampling of $q$ vectors. For the $\Gamma \rightarrow \mathrm{K}_{4}$ path, the $12 \times 12 \times 2$ grid naturally accommodates the $\mathrm{K}_{1}\left(\frac{1}{3} \frac{1}{3} 0\right)$ and $\mathrm{K}_{4}\left(\frac{2}{3} \frac{2}{3} 0\right)$ points. The pertaining Kohn-Sham structure (KSS) and screening files were then constructed using 100 total bands, with a total cutoff energy of $20 \mathrm{Ha}$. The final KSS file includes a shift of $(0.1,0.2,0.3)$ in order to improve convergence over the total number of $\mathbf{k}$ points.

Lastly, we used the DP/EXC code $[66,67]$ to execute the calculation of the macroscopic dielectric function $(\epsilon)$ and the EEL spectra for different values of $q$. We obtained converged spectra using 12 shells of reciprocal-space vectors $(\mathbf{G}), 20$ shells of plane waves, and 80 total bands for all methods, except when explicitly noted otherwise. Local-field effects have been taken into account in every calculation presented here. The macroscopic dielectric functions and EEL spectra have a $0.6 \mathrm{eV}$ Gaussian broadening applied to them. Furthermore, in order to simulate the band-structure stretching obtained from $G W$ results [68], a stretching of $10 \%$ was applied to the valence-band energies, and 5\% to the conduction band energies. These parameters and corrections were kept uniform across all methods studied here. 400 iterations were used for calculations that make use of the Haydock iterative method $[15,22,28-30,61]$.

The DP/EXC code was enabled to use the PETSc [69] and SLEPc [70] libraries for diagonalizing [71] the complete excitonic Hamiltonian using MPI parallelism. In fact, it is the inclusion of these libraries that makes it possible to solve the BSE CP (with coupling terms included) in a reasonable amount of time. All other calculations use the OpenMP parallel API, for efficient parallelization and memory use on a single machine. The Supplemental Material [72] contains detailed computational benchmarks concerning these calculations.

\section{RESULTS}

We calculate the macroscopic dielectric functions and the subsequent EEL spectra over an energy range between 0 to $15 \mathrm{eV}$, enough to cover the dispersion behavior of the $\pi$ plasmon for each value of momentum transfer $q$. Figure 1 depicts the two selected in-plane $q$ paths along which we calculate our results. The $\Gamma \rightarrow \mathrm{M}_{4}$ path has only variation of $q_{x}$, and passes through the $\mathrm{M}$ point of the first zone. The $\Gamma \rightarrow \mathrm{K}_{4}$ path varies both $q_{x}$ and $q_{y}$ and passes through the $\mathrm{K}$ point of the first zone and the $\mathrm{M}$ point of the third zone. As mentioned in Sec. III, we used $14 \times 14 \times 02$ and $12 \times 12 \times 02 \mathbf{k}$-point grids for these calculations, allowing us to evaluate the $q$ dependence in steps of $1 / 14$ and/or $1 / 12$ for each path. Shared points between these two grids $\left(\Gamma, M_{1}\right.$, and $\mathrm{M}_{4}$ ) are virtually identical, meaning that both grids have acceptable convergence.

A plasmon excitation is identified as the point where $\operatorname{Re} \epsilon_{\mathrm{M}}=0$ (crossing from negative to positive), and $\operatorname{Im} \epsilon_{\mathrm{M}}$ is nearly zero. For convenience, we will refer to this as the plasmon criterion [73]; therefore, peaks in the EEL spectra that meet this condition will naturally be understood to be plasmon excitations. The general physical picture of the $q$-dependent plasmon dispersion is as follows. The shape and intensity of $\operatorname{Re} \epsilon_{\mathrm{M}}(\mathbf{q}, \omega)$ are indicative of the electron screening behavior, and $\operatorname{Im} \epsilon_{\mathrm{M}}(\mathbf{q}, \omega)$ is indicative of interband transitions [65]. Plasmonic behavior for small values of $q$ are present in the EEL spectrum, with the thin peaked $\pi$ plasmon appearing around $7 \mathrm{eV}$. For values of $q<1.0 \AA^{-1}$, the plasmon criterion is met and the plasmon peak occurs very close to the point where $\operatorname{Re} \epsilon_{\mathrm{M}}(\mathbf{q}, \omega)$ is zero. This holds true for both the $\Gamma \rightarrow \mathrm{M}$, and $\Gamma \rightarrow \mathrm{K}$ directions. For larger values of $q$, the plasmon criterion is no longer met, as $\operatorname{Re} \epsilon_{\mathrm{M}}(\mathbf{q}, \omega)$ takes on only positive values; however, the EEL spectrum still presents defined peaks that correspond to interband transitions only [65]. This is in accordance to Eq. (3), where the EEL spectra will begin to be less and less affected by the reduced screening of $\operatorname{Re} \epsilon_{\mathrm{M}}(\mathbf{q}, \omega)$, and essentially mimic $\operatorname{Im} \epsilon_{\mathrm{M}}(\mathbf{q}, \omega)$.

In Fig. 2, we present a general comparison of the EEL spectra and macroscopic dielectric function between the different methods, centered around the energy range of the $\pi$ plasmon. For this low value of $q=0.21 \AA^{-1}$, the peak in the EEL spectra meets the aforementioned plasmon criterion and the plasmon peak energy position matches the zero crossing (from negative to positive) of $\operatorname{Re} \epsilon_{\mathrm{M}}(\mathbf{q}, \omega)$. $\operatorname{Im} \epsilon_{\mathrm{M}}(\mathbf{q}, \omega)$ (not shown) is also very small around this transition point. The plasmon peak is present for all calculated methods; however, peak position and intensity vary significantly between them. The predominant difference in peak intensity comes from the inclusion of the coupling terms in the excitonic Hamiltonian. Considering the applied broadening, methods that include these coupling terms (BSE CP, ALDA CP, and RPA CP) have peak intensities that are closer to experiment $[43,45,65]$; the remaining methods produce peaks that are roughly twice as 

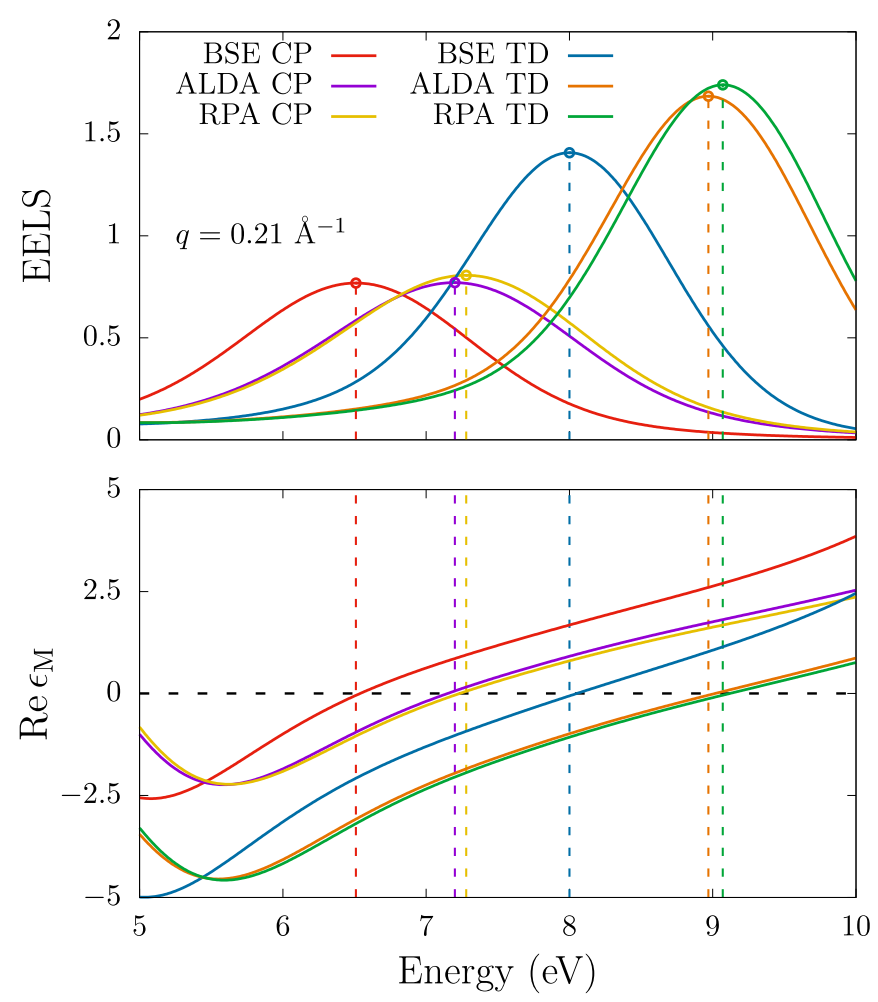

FIG. 2. Top panel: Comparison of the different theoretical EEL spectra in the $\pi$-plasmon region at the same momentum transfer $q=0.21 \AA^{-1}$. Bottom panel: The corresponding real part of the dielectric function, $\operatorname{Re} \epsilon_{\mathrm{M}}$. The plasmon criterion is met for this low value of $q$; however, the plasmon peak position and intensity vary widely between each method.

intense. The ALDA and RPA calculations yield spectra that are generally quite similar. We will discuss the peak positions in more detail below when addressing the $q$-dependent plasmon dispersion.

Figure 3 depicts the differences introduced into both the EEL spectrum and the dielectric function when applying valence (conduction) band stretching of $10 \%$ (5\%). As mentioned above, this band stretching is taken from $G W$ results [68] and compares very well with photoemission measurements $[65,74]$. Both the EEL spectrum and dielectric function are shifted towards higher energies; however, the nature of the stretching causes a redistribution of the band energies, which causes changes in both the peak position and intensities. Since these values are applied to the DFT band energies that are used subsequently in the calculation of the dielectric function, this behavior is consistent across all methods, and for all calculated values of $q$. This stretching improves the agreement of the peak positions between the calculated and the experimental EELS spectra.

In Fig. 4, we present the $q$-dependent dispersion behavior of the $\pi$ plasmon, for values of $q$ along the $\Gamma \rightarrow \mathrm{M}_{1}$ path. Colored points represent the different calculations, while the black squares are data points from high-resolution EELS measurements reproduced with permission from Ref. [51]. The peaks in the EEL spectra split into two branches: a main branch that is present for all values of $q$ with energy values between 7 and $13 \mathrm{eV}$, and a secondary branch that begins to
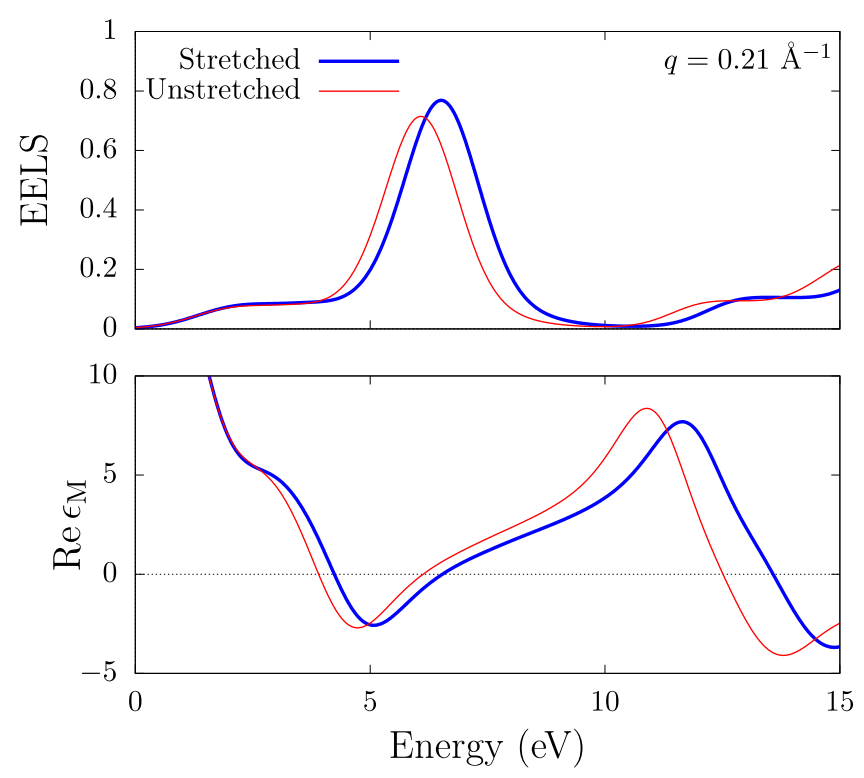

FIG. 3. Top panel: EEL spectra calculated with the BetheSalpeter equation, including the coupling terms in the excitonic Hamiltonian (BSE CP), for a transferred momentum $q=0.21 \AA^{-1}$, calculated with both stretched and unstretched valence and conduction bands. Bottom panel: The corresponding real part of the macroscopic dielectric function, $\operatorname{Re} \epsilon_{\mathrm{M}}$. The band stretching causes the spectra peaks to shift nonrigidly to higher energies.

appear after $q=0.6 \AA^{-1}$ with energy values between 5 and $8 \mathrm{eV}$. As mentioned above, not all of these points represent plasmon excitations; in general, points for values of $q>$ $1.0 \AA^{-1}$ do not meet the plasmon criterion and are primarily produced by interband transitions [65]. However, these points are measured by EELS experiments and are therefore included in our comparison.

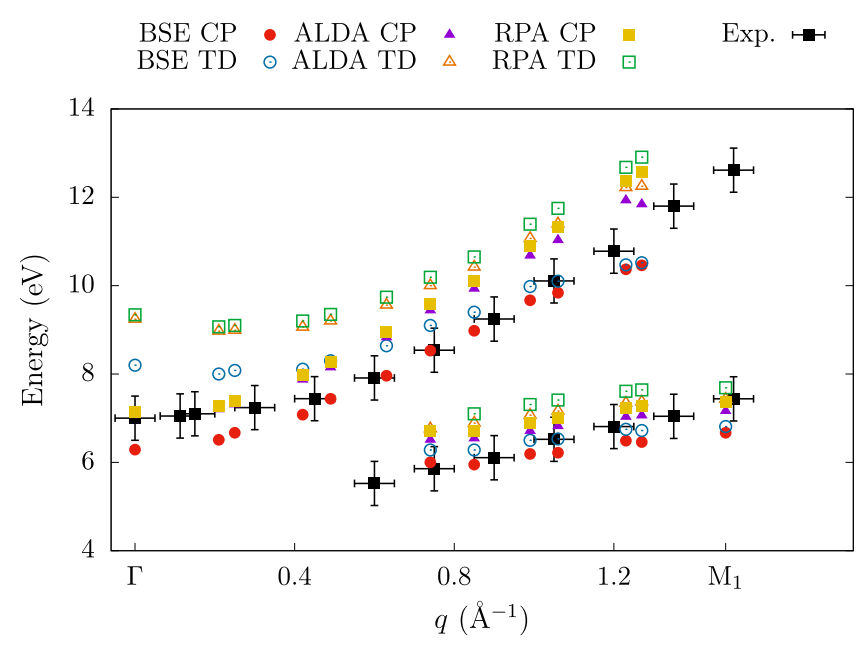

FIG. 4. The $\pi$-plasmon dispersion, obtained by plotting the peak maximum (in $\mathrm{eV}$ ) as a function of transferred momentum $q$ (along the $\Gamma \rightarrow M_{1}$ path). The peak positions calculated using each theoretical method are compared with experimental data from Ref. [51]. The uneven spacing between the calculated points is due to the use of both the $12 \times 12 \times 2$ and $14 \times 14 \times 2 \mathbf{k}$-point grids. 


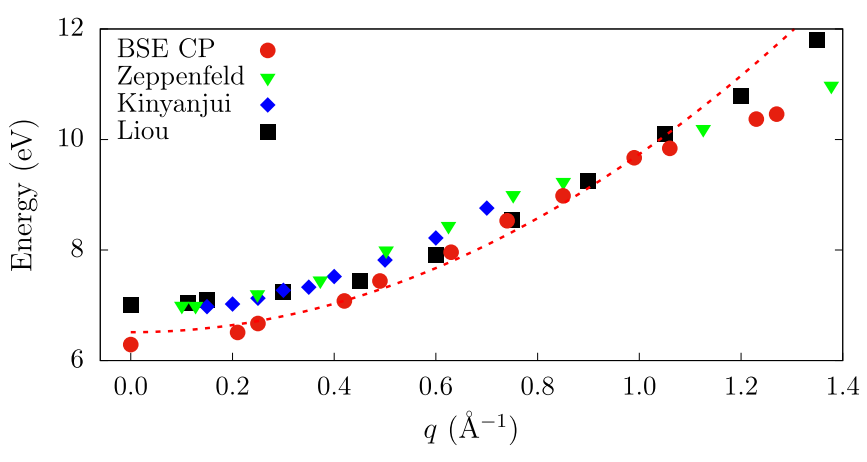

FIG. 5. A more detailed view of the $\pi$-plasmon peak dispersion along the $\Gamma \rightarrow \mathrm{M}_{1}$ path; "BSE CP" is our theoretical result (featured in Fig. 4), "Zeppenfeld" is experimental data from Ref. [43], "Kinyanjui" from Ref. [50], and "Liou" from Ref. [51]. Error bars have been excluded from the "Liou" data for improved legibility. The red dashed line is a quadratic fit for the theoretical points.

It is evident from the plot that the peak positions vary widely across all methods, and some general trends can be seen. Calculations that employ the TDA are generally shifted towards higher energies over their counterparts; thus, ALDA TD and RPA TD typically present the least similarity with the experimental data. ALDA CP and RPA CP are quite similar, with ALDA CP presenting some improvement over RPA CP. Both of these methods tend to have a higher energy value than the experiment, although they fit well for low values of $q$. The BSE CP calculation coincides well with the experimental points throughout the entire $q$ range, but tends to underestimate the energy for large values of $q$. Both BSE calculations begin to coincide as the value of $q$ increases.

In Fig. 5, we focus on the upper branch and compare our most complete calculation, BSE CP, against three separate experimental EELS measurements taken from Refs. [43], [50], and [51]. These experiments are mostly consistent with each other across the range of transferred momentum. Our calculation yields, in general, quantitatively similar results to the measured peak values. All of our calculations follow the well-documented quadratic dispersion relation $[43,65]$; in particular, the BSE CP method follows this relation for values of $q<1.2 \AA^{-1}$. The red dashed line in the figure is a quadratic fit for the theoretical points. Overall, our calculated results can be directly compared with experiments that span almost five decades, taken by completely different groups.

These trends are in accordance with the approximations implied for each method. First, taking into account excitons (e-h interactions) causes the peak position to shift towards lower energies [25]; second, the inclusion of the coupling terms in the Hamiltonian further shifts the peak position to lower energies [36,37]. This explains why BSE CP has the lowest peak energies, and why RPA TD and ALDA TD have the highest. Furthermore, the TDA is known to fail at describing plasmonic excitations [34-37] due to the neglected e-h antipairs; thus, for small values of $q$, BSE TD tends to overestimate the peak energy. However, as the momentum transfer increases the plasmon dissipates into interband transitions (reducing the number of e-h antipairs), and the BSE $\mathrm{CP}$ and BSE TD calculations begin to coincide. In fact, this behavior holds true for all methods; in other words, the TDA
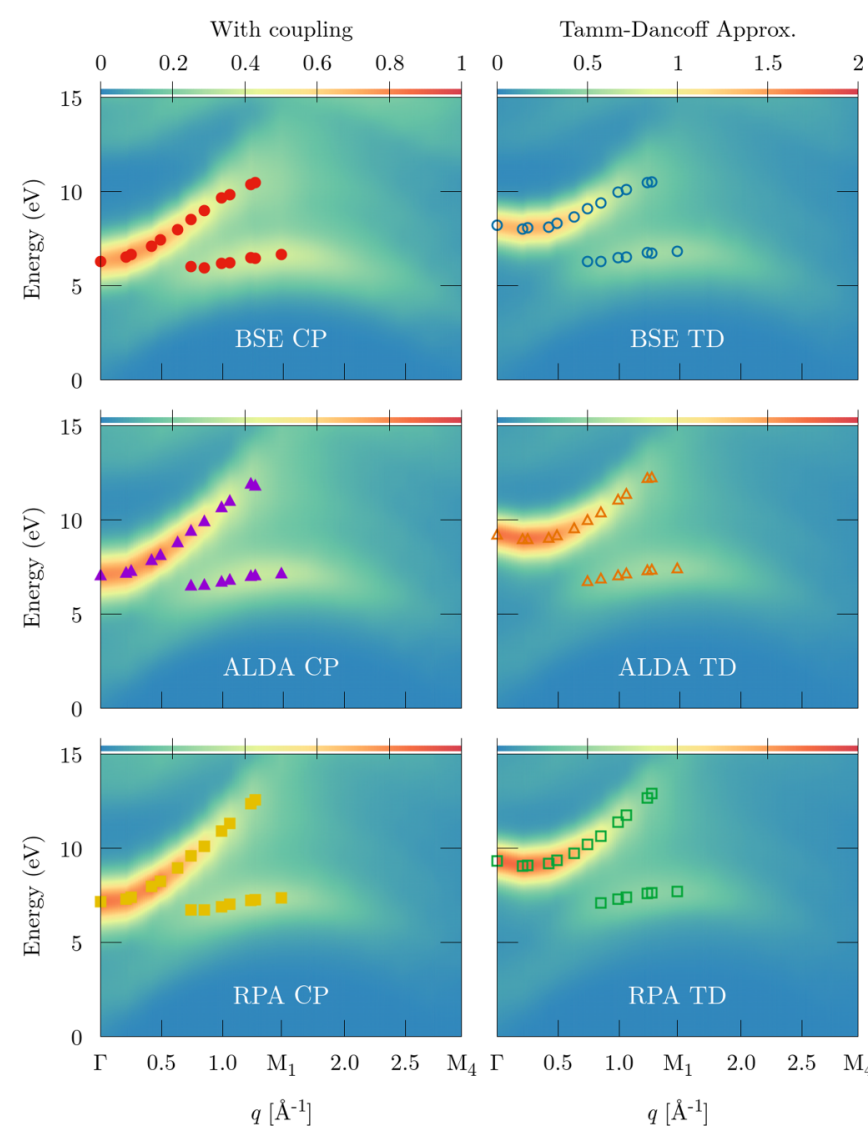

FIG. 6. Color map representations of the calculated EEL spectra vs transferred momentum ( $q$ ) for the $\pi$-plasmon region, for $q$ values along the $\Gamma \rightarrow \mathrm{M}_{4}$ path. Methods including the coupling terms in the excitonic Hamiltonian are shown in the left column, and methods neglecting these terms (Tamm-Dancoff approximation) are shown in the right column. The EEL intensity is represented by the color palette ranging from blue to red. The exact peak positions from Fig. 4 are superimposed on each color map.

can accurately describe the peak positions when plasmon behavior is not expected, or there is little to no contribution from e-h antipairs.

In order to elucidate the general trends beyond the experimental data range (past the $\mathrm{M}_{1}$ point in the first Brillouin zone), we extend our calculations all the way up to the fourth Brillouin zone to the $\mathrm{M}_{4}$ point. Figure 6 depicts the complete plasmon dispersion for each calculation, for $q$ values along the $\Gamma \rightarrow M_{4}$ path. The left side of the figure presents the calculated dispersion including the coupling terms, and the right side with calculations that neglect them; the EELS intensity scale is located above each column. The peak positions featured in Fig. 4 are superimposed over each color map. All methods agree that the upper branch mostly dissipates for $q$ values beyond $\mathrm{M}_{1}\left(1.48 \AA^{-1}\right)$, while the lower branch continues to exist throughout the range. As mentioned above, the behavior of both $\epsilon_{1}$ and $\epsilon_{2}$, which does not conform to the plasmon criterion for these high $q$ values, indicates that these peaks are primarily due to interband transitions and are not plasmons. Our predictions also show that the lower branch presents negative dispersion after $\mathrm{M}_{1}$, and appears to return to lower energies rather than continuing upwards. Confirmation 


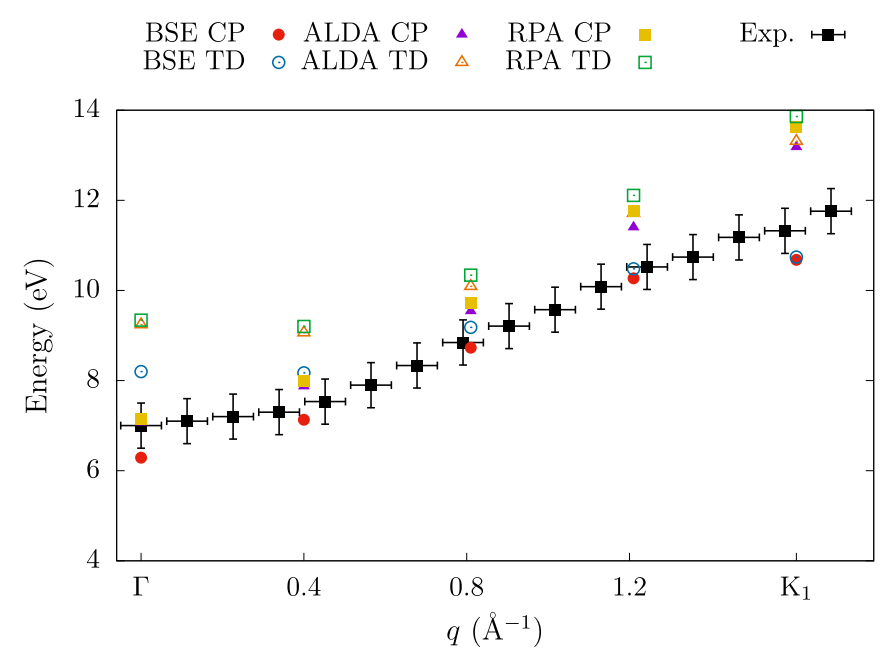

FIG. 7. The $\pi$-plasmon dispersion, obtained by plotting the peak maximum (in $\mathrm{eV}$ ) as a function of transferred momentum $q$ (along the $\Gamma \rightarrow \mathrm{K}_{1}$ path). The peak positions calculated using each theoretical method are compared with experimental data from Ref. [51].

of this trend will require future experimental measurements taken at similarly high values of $q$. While these plots are very useful to discern trends, they show that the differences between each method are subtle, and must be analyzed in closer detail as we have done in Figs. 4 and 5.

Likewise, we conduct a similar analysis for the $\Gamma \rightarrow \mathrm{K}_{1}$ path in Fig. 7. For these values of $q$, the spectra are dominated by a single peak with energy positions ranging from 7 to $12 \mathrm{eV}$, where the classical plasmonic behavior is lost after $q \approx 1.00 \AA^{-1}$. Once again, our calculations confirm the strong dispersion of the peak position. ALDA TD and RPA TD significantly overestimate the peak energy position, and $\mathrm{CP}$ and TD calculations begin to coincide for increasing momentum transfer. BSE calculations yield good agreement with experiment throughout the range. We also extend our calculations for increasing $q$ values into the fourth Brillouin zone $\mathrm{K}_{4}$ point.

The Supplemental Material [72] features a similar analysis of the $\pi+\sigma$ plasmon that occurs in the $25-45 \mathrm{eV}$ energy range. We review the trends of each theoretical method, as there are few experimental data available for this energy range.

\section{CONCLUSIONS}

We carried out a systematic study of the macroscopic dielectric function and EEL spectra for the $\pi$ plasmon of graphite, comparing ab initio TDDFT and BSE methods. We selected graphite as a good benchmark case due to the extensive theoretical and experimental work available. Our results coincide with previous literature (where available), and offer quantitative agreement with experiment. Using two nonequivalent momentum paths spanning the first four Brillouin zones, we demonstrate the importance of including the coupling terms in the excitonic Hamiltonian for describing plasmon excitations. The TDA, while numerically efficient, cannot reproduce some key features in the experimental EEL spectra. Access to high-resolution EELS measurements around the $\pi$ plasmon allow us to compare the detailed behavior and trends of each method.

Overall, we provide an overview of $a b$ initio methods that are considered state-of-the-art at describing the optoelectronic properties of solid-state systems. We have applied these methods on graphite, which offers an excellent benchmark material for this type of study. We are confident this study can be used as a reference for future work. The knowledge of the inverse dielectric function for the wide range of momentum transfer (beyond the experimental range available today) that we provide gives useful insights also for other methods which are intrinsically based on the concept of Coulomb screening, like many-body $G W$ (and beyond $G W$ ) approaches [57,75], hybrid functionals in DFT [76], or dynamical mean field theory [77].

Finally, we present real-world computational benchmarks in the Supplemental Materials [72]; as these clearly show, the significant computational cost of BSE CP may not be necessarily warranted for every situation. The very low computational expense of the TDDFT methods compared to the BSE makes them an attractive alternative when the coupling terms are the determining factors for a calculation. In summary, these methods should always be applied judiciously and their use evaluated on a case-by-case basis.

\section{ACKNOWLEDGMENTS}

The authors would like to thank S. C. Liou for graciously contributing the experimental data featured in this work, and also M. Gatti and I. Reshetnyak for fruitful discussion. The authors thankfully acknowledge the computer resources, technical expertise, and support provided by the Laboratorio Nacional de Supercómputo del Sureste de México, a member of the CONACYT network of national laboratories. S.M.A. acknowledges partial support for this project from CONACYT-México Scholarship No. 349278. B.S.M. acknowledges funding from CONACYT-Mexico Research Project No. A1-S-9410.
[1] P. Hohenberg and W. Kohn, Phys. Rev. 136, B864 (1964).

[2] W. Kohn and L. J. Sham, Phys. Rev. 140, A1133 (1965).

[3] E. Runge and E. K. U. Gross, Phys. Rev. Lett. 52, 997 (1984).

[4] G. Onida, L. Reining, and A. Rubio, Rev. Mod. Phys. 74, 601 (2002).

[5] V. I. Gavrilenko and F. Bechstedt, Phys. Rev. B 55, 4343 (1997).

[6] A. L. Fetter and J. D. Walecka, Quantum Theory of Many Particle Systems (McGraw-Hill, Inc., New York, 1972).
[7] L. Hedin, Phys. Rev. 139, A796 (1965).

[8] E. E. Salpeter and H. A. Bethe, Phys. Rev. 84, 1232 (1951).

[9] A. A. Abrikosov, L. P. Gorlcov, and I. E. Illyaloslinski, Methods of Quantum Field Theory in Statistical Physics, Quantum Field Theoretical Methods in Statistical Physics, Vol. 4 (Pergamon Press, Oxford, 1965).

[10] L. J. Sham and T. M. Rice, Phys. Rev. 144, 708 (1966).

[11] W. Hanke and L. J. Sham, Phys. Rev. B 21, 4656 (1980). 
[12] E. L. Shirley and S. G. Louie, Phys. Rev. Lett. 71, 133 (1993).

[13] S. Albrecht, G. Onida, and L. Reining, Phys. Rev. B 55, 10278 (1997).

[14] L. X. Benedict, E. L. Shirley, and R. B. Bohn, Phys. Rev. Lett. 80, 4514 (1998).

[15] L. X. Benedict and E. L. Shirley, Phys. Rev. B 59, 5441 (1999).

[16] L. X. Benedict, A. Puzder, A. J. Williamson, J. C. Grossman, G. Galli, J. E. Klepeis, J.-Y. Raty, and O. Pankratov, Phys. Rev. B 68, 085310 (2003).

[17] M. Palummo, O. Pulci, R. Del Sole, A. Marini, P. Hahn, W. G. Schmidt, and F. Bechstedt, J. Phys.: Condens. Matter 16, S4313 (2004).

[18] A. Sitt, L. Kronik, S. Ismail-Beigi, and J. R. Chelikowsky, Phys. Rev. A 76, 054501 (2007).

[19] L. E. Ramos, J. Paier, G. Kresse, and F. Bechstedt, Phys. Rev. B 78, 195423 (2008).

[20] D. Rocca, D. Lu, and G. Galli, J. Chem. Phys. 133, 164109 (2010).

[21] J. M. Garcia-Lastra, J. D. Bass, and K. S. Thygesen, J. Chem. Phys. 135, 121101 (2011).

[22] M. Grüning, A. Marini, and X. Gonze, Comput. Mater. Sci 50, 2148 (2011).

[23] M. Gatti and F. Sottile, Phys. Rev. B 88, 155113 (2013).

[24] G. Onida, L. Reining, R. W. Godby, R. Del Sole, and W. Andreoni, Phys. Rev. Lett. 75, 818 (1995).

[25] S. Albrecht, L. Reining, R. Del Sole, and G. Onida, Phys. Rev. Lett. 80, 4510 (1998)

[26] L. X. Benedict, E. L. Shirley, and R. B. Bohn, Phys. Rev. B 57, R9385 (1998).

[27] M. Rohlfing and S. G. Louie, Phys. Rev. Lett. 81, 2312 (1998).

[28] R. Haydock, V. Heine, and M. J. Kelly, J. Phys. C: Solid State Phys. 5, 2845 (1972).

[29] R. Haydock, Comput. Phys. Commun. 20, 11 (1980).

[30] D. Rocca, R. Gebauer, Y. Saad, and S. Baroni, J. Chem. Phys. 128, 154105 (2008).

[31] M. Lopez del Puerto, M. L. Tiago, and J. R. Chelikowsky, Phys. Rev. Lett. 97, 096401 (2006).

[32] B. Arnaud, S. Lebègue, P. Rabiller, and M. Alouani, Phys. Rev. Lett. 96, 026402 (2006).

[33] L. Wirtz, A. Marini, and A. Rubio, Phys. Rev. Lett. 96, 126104 (2006).

[34] R. Zimmermann, Phys. Status Solidi B 41, 23 (1970).

[35] W. A. Caliebe, J. A. Soininen, E. L. Shirley, C.-C. Kao, and K. Hämäläinen, Phys. Rev. Lett. 84, 3907 (2000).

[36] V. Olevano and L. Reining, Phys. Rev. Lett. 86, 5962 (2001).

[37] M. Grüning, A. Marini, and X. Gonze, Nano Lett. 9, 2820 (2009).

[38] F. Bassani and G. P. Parravicini, Nuovo Cimento B 50, 95 (1967).

[39] G. S. Painter and D. E. Ellis, Phys. Rev. B 1, 4747 (1970).

[40] A. Grüneis, C. Attaccalite, T. Pichler, V. Zabolotnyy, H. Shiozawa, S. L. Molodtsov, D. Inosov, A. Koitzsch, M. Knupfer, J. Schiessling, R. Follath, R. Weber, P. Rudolf, L. Wirtz, and A. Rubio, Phys. Rev. Lett. 100, 037601 (2008).

[41] F. Matsui, H. Nishikawa, H. Daimon, M. Muntwiler, M. Takizawa, H. Namba, and T. Greber, Phys. Rev. B 97, 045430 (2018)

[42] E. A. Taft and H. R. Philipp, Phys. Rev. 138, A197 (1965).

[43] K. Zeppenfeld, Z. Phys. A: Hadrons Nucl. 243, 229 (1971).

[44] H. Venghaus, Phys. Status Solidi B 66, 145 (1974).
[45] U. Büchner, Phys. Status Solidi B 81, 227 (1977).

[46] A. G. Marinopoulos, L. Reining, V. Olevano, A. Rubio, T. Pichler, X. Liu, M. Knupfer, and J. Fink, Phys. Rev. Lett. 89, 076402 (2002).

[47] C. Kramberger, R. Hambach, C. Giorgetti, M. H. Rümmeli, M. Knupfer, J. Fink, B. Büchner, L. Reining, E. Einarsson, S. Maruyama, F. Sottile, K. Hannewald, V. Olevano, A. G. Marinopoulos, and T. Pichler, Phys. Rev. Lett. 100, 196803 (2008).

[48] M. F. Lin, C. S. Huang, and D. S. Chuu, Phys. Rev. B 55, 13961 (1997).

[49] P. E. Trevisanutto, M. Holzmann, M. Côté, and V. Olevano, Phys. Rev. B 81, 121405(R) (2010).

[50] M. K. Kinyanjui, C. Kramberger, T. Pichler, J. C. Meyer, P. Wachsmuth, G. Benner, and U. Kaiser, Europhys. Lett. 97, 57005 (2012).

[51] S. C. Liou, C.-S. Shie, C. H. Chen, R. Breitwieser, W. W. Pai, G. Y. Guo, and M.-W. Chu, Phys. Rev. B 91, 045418 (2015).

[52] L. Yang, J. Deslippe, C.-H. Park, M. L. Cohen, and S. G. Louie, Phys. Rev. Lett. 103, 186802 (2009).

[53] C. Tegenkamp, H. Pfnür, T. Langer, J. Baringhaus, and H. W. Schumacher, J. Phys. Condens. Matter 23, 012001 (2011).

[54] A. Politano and G. Chiarello, Nanoscale 6, 10927 (2014).

[55] L. G. Bulusheva, O. V. Sedelnikova, and A. V. Okotrub, Int. J. Quantum. Chem. 116, 270 (2016).

[56] P. Li, X. Ren, and L. He, Phys. Rev. B 96, 165417 (2017).

[57] D. M. Ceperley, L. Reining, and R. M. Martin, Interacting Electrons: Theory and Computational Approaches (Cambridge University Press, Cambridge, 2016).

[58] F. Sottile, V. Olevano, and L. Reining, Phys. Rev. Lett. 91, 056402 (2003).

[59] M. Rohlfing and S. G. Louie, Phys. Rev. B 62, 4927 (2000)

[60] F. Sottile, Response functions of semiconductors and insulators: From the Bethe-Salpeter equation to time-dependent density functional theory, Ph.D. thesis, Ecole Polytechnique X, 2003.

[61] M. P. Ljungberg, P. Koval, F. Ferrari, D. Foerster, and D. Sánchez-Portal, Phys. Rev. B 92, 075422 (2015).

[62] N. Troullier and J. L. Martins, Phys. Rev. B 43, 1993 (1991).

[63] X. Gonze, B. Amadon, P.-M. Anglade, J.-M. Beuken, F. Bottin, P. Boulanger, F. Bruneval, D. Caliste, R. Caracas, M. Côté, T. Deutsch, L. Genovese, P. Ghosez, M. Giantomassi, S. Goedecker, D. R. Hamann, P. Hermet, F. Jollet, G. Jomard, S. Leroux, M. Mancini, S. Mazevet, M. J. T. Oliveira, G. Onida, Y. Pouillon, T. Rangel, G.-M. Rignanese, D. Sangalli, R. Shaltaf, M. Torrent, M. J. Verstraete, G. Zerah, and J. W. Zwanziger, Comput. Phys. Commun. 180, 2582 (2009).

[64] The ABINIT code is a common project of the Université Catholique de Louvain, Corning Incorporated, and other contributors (http://www.abinit.org).

[65] A. G. Marinopoulos, L. Reining, A. Rubio, and V. Olevano, Phys. Rev. B 69, 245419 (2004).

[66] V. Olevano, L. Reining, and F. Sottile, http://dp-code.org.

[67] L. Reining, V. Olevano, F. Sottile, S. Albrecht, and G. Onida, http://www.bethe-salpeter.org.

[68] V. Olevano (private communication).

[69] S. Balay, S. Abhyankar, M. F. Adams, J. Brown, P. Brune, K. Buschelman, L. Dalcin, V. Eijkhout, W. D. Gropp, D. Kaushik, M. G. Knepley, D. A. May, L. Curfman McInnes, K. Rupp, 
B. F. Smith, S. Zampini, H. Zhang, and H. Zhang, PETSc web page, http://www.mcs.anl.gov/petsc.

[70] V. Hernandez, J. E. Roman, and V. Vidal, ACM Trans. Math. Software 31, 351 (2005).

[71] I. Reshetnyak, Computing optical properties and photoemission spectra: A new starting point, Ph.D. thesis, Ecole Polytechnique, 2015.

[72] See Supplemental Material at http://link.aps.org/supplemental/ 10.1103/PhysRevB.100.045205 for a detailed convergence report of the RPA calculation, a predictive analysis of the dispersion behavior of the $\pi+\sigma$ plasmon, and real-world computational benchmarks for each theoretical method included in this work.
[73] W. Jones and N. H. March, Theoretical Solid State Physics, Vol. 1: Perfect Lattices in Equilibrium, Interscience Monographs and Texts in Physics and Astronomy, Vol. 1 (WileyInterscience, London, 1973).

[74] C. Heske, R. Treusch, F. J. Himpsel, S. Kakar, L. J. Terminello, H. J. Weyer, and E. L. Shirley, Phys. Rev. B 59, 4680 (1999).

[75] C. Rödl, K. O. Ruotsalainen, F. Sottile, A.-P. Honkanen, J. M. Ablett, J.-P. Rueff, F. Sirotti, R. Verbeni, A. Al-Zein, L. Reining, and S. Huotari, Phys. Rev. B 95, 195142 (2017).

[76] A. V. Krukau, O. A. Vydrov, A. F. Izmaylov, and G. E. Scuseria, J. Chem. Phys. 125, 224106 (2006).

[77] S. Biermann, J. Phys.: Condens. Matter 26, 173202 (2014). 